\title{
Pulmonary alveolar proteinosis and Mycobacterium abscessus lung infection related to ruxolitinib after allogeneic stem cell transplantation
}

To the Editor:

Non-infectious pulmonary complications are frequent after allogeneic haematopoietic stem cell transplantation (HSCT). They mainly comprise bronchiolitis obliterans and interstitial pneumonia in the setting of chronic graft-versus-host disease (cGVHD) [1].

Pulmonary alveolar proteinosis (PAP) is a rare disorder characterised by accumulation of lipoproteinaceous material in alveolar spaces due to alveolar macrophage dysfunction [2]. It has been described as a rare early complication after HSCT [3]. We report the case of a patient who developed PAP associated with Mycobacterium abscessus infection several years after an allogeneic HSCT. This case is very unusual because of its late onset. We suggest these manifestations were drug-induced, related to the functional deficiency of the alveolar macrophages induced by ruxolitinib.

A 66-year-old female was diagnosed with a myelodysplasic syndrome with blastic transformation in 2011. After having received daunorubicin and azacitidine, she underwent a sibling donor HSCT in January 2013. The conditioning regimen consisted of fludarabine, busulfan and anti-thymocyte globulins.

She developed severe chronic cutaneous cGVHD from August 2013. Several therapies were unsuccessful, until ruxolinitib (20 mg per day) was added in October 2015. The extensive sclerodermic cutaneous cGVHD was stabilised in 6 months with a combination of oral corticosteroids (15 mg per day), ciclosporin A, methotrexate, extracorporeal photochemotherapy and ruxolitinib. Anti-infectious prophylaxis consisted of posaconazole, valaciclovir, trimethoprim-sulfamethoxazole and azithromycin.

In May 2016, she began to complain of slowly increasing non-febrile dyspnoea. Pulmonary function tests (PFTs) revealed normal values except for a recent decrease in diffusing capacity of the lung for carbon monoxide capacity (DLCO) to $45 \%$ of the predicted value. A thoracic computed tomography (CT) scan revealed bilateral ground-glass opacities predominant in the upper lobes and thickened interlobular septa. Bronchoalveolar lavage (BAL) fluid analysis displayed 50000 cells per $\mathrm{mL}$, with $88 \%$ macrophages, $8 \%$ lymphocytes and $4 \%$ neutrophils. Specific stains (Ziehl, Grocott and Periodic acid-Schiff (PAS)) and extensive microbiological tests were negative. Despite methotrexate interruption and antibiotic therapy, pulmonary infiltrates extended on the thoracic CT scan.

In January 2017, video-assisted biopsies were performed on all three lobes of the right lung. Histological analysis showed an intra-alveolar accumulation of eosinophilic proteinaceous granular material, which stained positive with the PAS stain. This proteinaceous material was extensive but patchy, with areas of normal lung, without inflammation, granuloma, necrosis or interstitial fibrosis. The Ziehl stain demonstrated extracellular acid-fast bacilli in alveolar spaces. Cultures in liquid medium were positive after 6 days for M. abscessus. Susceptibility testing showed inducible resistance to clarithromycin (T28 sequovar) and usual weak sensitivity to imipenem and amikacin (minimum inhibitory concentrations of 32 and $16 \mathrm{mg} \cdot \mathrm{L}^{-1}$, respectively).

The patient worsened rapidly with oxygen requirement and fever. Blood analysis showed: haemoglobin $7.8 \mathrm{~g} \cdot \mathrm{dL}^{-1}$, neutrophils 5700 per $\mathrm{mm}^{3}$, lymphocytes 600 per $\mathrm{mm}^{3}$, platelets 75000 per $\mathrm{mm}^{3}$ and C-reactive

@ERSpublications

Ruxolitinib may induce pulmonary alveolar proteinosis and favour respiratory non-tuberculous mycobacterial infections http://ow.ly/bTXn30jogC8

Cite this article as: Salvator $\mathrm{H}$, Berti E, Catherinot E, et al. Pulmonary alveolar proteinosis and Mycobacterium abscessus lung infection related to ruxolitinib after allogeneic stem cell transplantation. Eur Respir J 2018; 51: 1701960 [https://doi.org/10.1183/13993003.01960-2017]. 
protein $30 \mathrm{mg} \cdot \mathrm{L}^{-1}$. A thoracic CT scan revealed diffuse ground-glass opacities, crazy paving and areas of pulmonary consolidation. Antibiotics against M. abscessus (imipenem $750 \mathrm{mg}$ three times daily, amikacin $600 \mathrm{mg}$ three times per week and azithromycin $500 \mathrm{mg}$ per day) were started. Because of increased cytopenia, ruxolinitib was withdrawn.

Chimerism remained $100 \%$ donor and a myelogram did not show any sign of myelodysplasia. No serum anti-granulocyte monocyte colony-stimulating factor (GM-CSF) antibodies were detected. There was no GATA2 mutation.

The clinical course was rapidly favourable. 6 days after admission, the fever had abated and the patient was weaned from oxygen.

We pursued antibiotics for 7 months and replaced ruxolitinib with mycophenolate mofetil from February 2017. 4 months later, she felt significantly better, her thoracic CT scan (figure 1) and PFTs were greatly improved and DLCO increased to $51 \%$ of the predicted value. Cutaneous GVHD worsened after ruxolitinib cessation, but only to a small extent. Respiratory improvement is maintained 1 year after PAP and mycobacterial infection management, with only discrete persistent radiological infiltrates on the thoracic CT scan.

We report, to our knowledge, the first case of a late-onset secondary PAP occurring after allogeneic HSCT and an unusual alveolar M. abscesssus infection, which may both be related to ruxolitinib.

Three distinct aetiological forms of PAP have been described: 1) congenital PAP, due to mutations in genes encoding surfactant proteins; 2) acquired PAP, which is characterised by the acquisition of anti-GM-CSF antibodies; and 3) secondary PAP associated with various conditions including myeloid malignancies.

Although PAP occurring after allogeneic HSCT is well known, the case of our patient exhibits a number of interesting features.

1) Previous reports described PAP as an event occurring early after HSCT, before the immune reconstitution is effective $[4,5]$. By contrast, the PAP in our patient occurred more than 3 years after
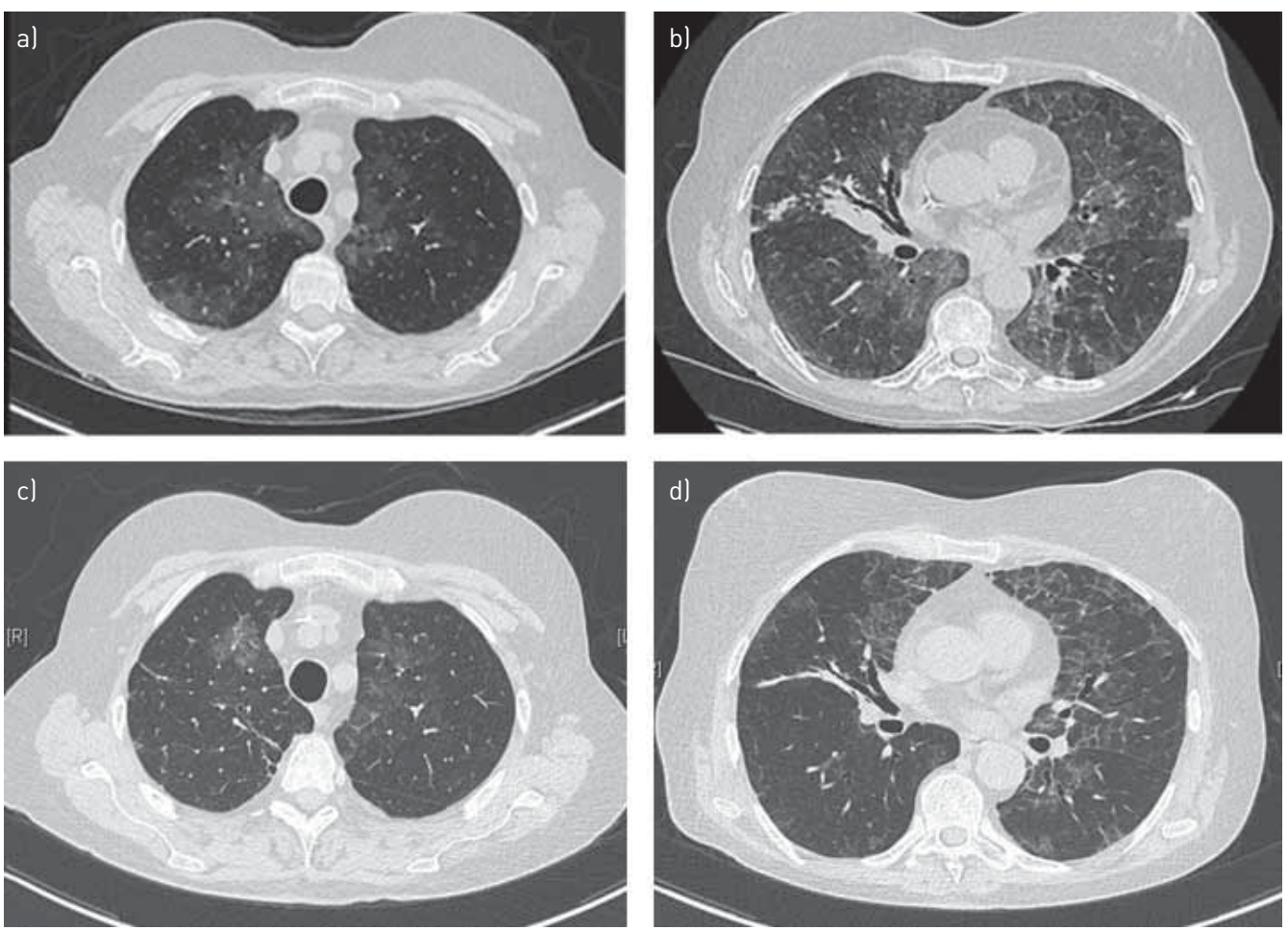

FIGURE $1 \mathrm{a}$, b) Computed tomography scan at the time of pulmonary alveolar proteinosis and mycobacterial infection diagnosis, highlighting ground-glass opacities, thickened interlobular septa and patchy condensations. c, d) Radiological evolution 4 months after beginning anti-mycobacterial treatment and withdrawal of ruxolitinib. 
allogeneic HSCT. One case of late-onset PAP occurring 18 months after HSCT had been previously reported, but only partially explored because of untimely death [6].

2) PAP diagnosis required surgical lung biopsy. Negativity of PAS staining on BAL fluid has previously been noted in reports of post-HSCT PAP [6].

3) None of the usual causes of PAP were detected. No anti-GM-CSF antibodies were detected. We eliminated a relapse of the haematological malignancy and GATA2 deficiency, evoked because of the association with alveolar proteinosis, myelodysplasic disorder and mycobacterial infection. A role for M. abscessus infection in the occurrence of PAP seems unlikely, as pulmonary involvement occurred several months before the isolation of mycobacteria and the patient improved despite partially active antibiotic treatment.

This led us to suspect the role of ruxolitinib, introduced 6 months before the occurrence of these two complications. Ruxolitinib is a selective Janus kinase (JAK) $1 / 2$ inhibitor, approved for the treatment of myelofibrosis and refractory cGVHD [7, 8]. Interestingly, the GM-CSF receptor is coupled with a Janus Kinase. The first step of intracellular signalling in macrophages depends on JAK2 activation and STAT3 phosphorylation. By disrupting GM-CSF intracellular signalling, ruxolitinib may have led to the onset of alveolar proteinosis.

Another striking feature of this case is the occurrence of an alveolar M. abscessus infection without any granuloma in the interstitial tissue or bronchial wall. Nontuberculous mycobacteria infections are rare after allogeneic HSCT, with incidence varying between 0.4 and $4.9 \%$ of graft recipients [9]. Catheter-related infections are the most commonly encountered nontuberculous mycobacteria complications, followed by pulmonary and cutaneous infections [9]. Infections by mycobacteria are a known complication of PAP [10]. Alveolar macrophages have a dual role in the lung immune response: phagocytosing intra-alveolar pathogens and guiding adaptive immunity. They are deficient in PAP. Moreover, the abundance of a lipoproteinaceous substrate favours pathogen adhesion and growth within the alveolar space. In our case, bacilli were observed exclusively in alveolar spaces within lipoproteinaceous material. It could be related to the ability of $M$. abscessus to obtain energy from the degradation of host-derived lipids with numerous lipase-encoding genes [11]. According to these mechanisms, ruxolitinib may also have favoured the occurrence of M. abscessus infection. Ruxolitinib is known to be associated with an increased risk of pneumonia (incidence of $9 \%$ at 12 months and $16.4 \%$ at 48 months in myelofibrosis) [12] and Mycobacterium tuberculosis reactivation [13]. Inhibiting the JAK-STAT pathway may increase the risk of mycobacterial infection by impairing the production and response to interferon (IFN)- $\gamma$, the function of dendritic cells and by reducing tumour necrosis factor- $\alpha$ production which plays a crucial role in granuloma formation [14]. This hypothesis is supported by the fact that no granuloma was observed in the lung biopsy of our patient, as reported in HIV-infected patients with low $\mathrm{CD} 4^{+}$cell count or in patients with complete IFN- $\gamma$ receptor deficiency [15].

In conclusion, physicians should be aware that ruxolitinib may be associated with severe pulmonary complications. We suggest that all patients should have a thoracic CT scan before initiation of ruxolitinib and be monitored for respiratory manifestations. Although it is impossible to indicate precise guidelines, we think that ruxolitinib should be stopped in the case of severe pulmonary infection and/or PAP occurrence. It is important to report these respiratory manifestations to the health authorities for worldwide surveillance.

Hélène Salvator $^{1,2,3}$, Enora Berti ${ }^{1}$, Emilie Catherinot ${ }^{1}$, Elisabeth Rivaud ${ }^{1}$, Alexandre Chabrol ${ }^{1,2}$, Stephanie Nguyen $^{4}$, Leila Zemoura $^{5}$, Emilie Cardot ${ }^{6}$, Colas Tcherakian ${ }^{1,2,3}$ and Louis-Jean Couderc ${ }^{1,2,3}$ on behalf of The Foch Hospital Lung Immune Deficiencies Study Group

${ }^{1}$ Foch Hospital, Respiratory Diseases Dept, Suresnes, France. ${ }^{2}$ Versailles Saint Quentin University, Montignyle-Bretonneux, France. ${ }^{3}$ UPRES EA 220, Suresnes, France. ${ }^{4}$ APHP, Pitié-Salpétrière Hospital, Haematology Dept, Paris, France. ${ }^{5}$ Foch Hospital, Pathology Dept, Suresnes, France. ${ }^{6}$ Foch Hospital, Microbiology Dept, Suresnes, France.

Correspondence: Hélène Salvator Hopital Foch Service de Pneumologie, 40 rue Worth 92150 Suresnes, France. Email: h.salvator@hopital-foch.com

Received: Sept 262017 | Accepted after revision: March 122018

Acknowledgments: The authors thank all the members of Foch Hospital Lung Immune Deficiencies study group for their dedication and their daily work with patients. Thoracic surgery: Alain Chapelier, Edouard Sage, Pierre Bonnette, and Philippe Puyo; intensive care unit: François Parquin and Gregoire Trebbia; radiology: François Mellot, Axel Guth, Fabrice Staub and Marie-Laure Chabi; microbiology: Eric Farfour and Pierre Cahen.

Author Contributions: H. Salvator, E. Berti, E. Catherinot and L.J. Couderc analysed clinical data and wrote the manuscript; E. Rivaud, A. Chabrol and C. Tcherakian analysed data and reviewed the manuscript; S. Nguyen addressed patients and reviewed the manuscript; L. Zemoura performed pathological analyses and reviewed the manuscript; E. Cardot designed microbiological analyses and reviewed the manuscript. 
Conflict of interest: H. Salvator reports non-financial support from Oxyvie, grants from LVL medical and Cardif assistance, and non-financial support from Boehringer, outside the submitted work. E. Catherinot reports non-financial support from LVL medical and CSL Behring, outside the submitted work. E. Rivaud reports non-financial support from Chiesi and Boehringer, outside the submitted work. A. Chabrol reports non-financial support from Homeperf, LVL medical and Roche, outside the submitted work. C. Tcherakian reports personal fees and non-financial support from Novartis, Boehringer and Chiesi, outside the submitted work. L.J. Couderc reports personal fees and non-financial support from Boehringer Ingelheim, grants and personal fees from Novartis, grants from LVL and ZAMS, and non-financial support from Teva, outside the submitted work.

\section{References}

1 Guihot A, Becquemin M-H, Couderc L-J, et al. Telemetric monitoring of pulmonary function after allogeneic hematopoietic stem cell transplantation. Transplantation 2007; 83: 554-560.

2 Trapnell BC, Whitsett JA, Nakata K. Pulmonary alveolar proteinosis. N Engl J Med. 2003; 349: 2527-2539.

3 Cordonnier C, Fleury-Feith J, Escudier E, et al. Secondary alveolar proteinosis is a reversible cause of respiratory failure in leukemic patients. Am J Respir Crit Care Med. 1994; 149: 788-794.

4 Ansari M, Rougemont A-L, Le Deist F, et al. Secondary pulmonary alveolar proteinosis after unrelated cord blood hematopoietic cell transplantation. Pediatr Transplant 2012; 16: E146-E149.

5 Tomonari A, Shirafuji N, Iseki T, et al. Acquired pulmonary alveolar proteinosis after umbilical cord blood transplantation for acute myeloid leukemia. Am J Hematol 2002; 70: 154-157.

6 Pidala J, Khalil F, Fernandez H. Pulmonary alveolar proteinosis following allogeneic hematopoietic cell transplantation. Bone Marrow Transplant 2011; 46: 1480-1483.

7 Verstovsek S, Mesa RA, Gotlib J, et al. A double-blind, placebo-controlled trial of ruxolitinib for myelofibrosis. N Engl J Med 2012; 366: 799-807.

8 Zeiser R, Burchert A, Lengerke C, et al. Ruxolitinib in corticosteroid-refractory graft-versus-host disease after allogeneic stem cell transplantation: a multicenter survey. Leukemia 2015; 29: 2062-2068.

9 Al-Anazi KA, Al-Jasser AM, Al-Anazi WK. Infections caused by non-tuberculous mycobacteria in recipients of hematopoietic stem cell transplantation. Front Oncol 2014; 4: 311.

10 Punatar AD, Kusne S, Blair JE, et al. Opportunistic infections in patients with pulmonary alveolar proteinosis. J Infect 2012; 65: 173-179.

11 Ripoll F, Pasek S, Schenowitz C, et al. Non mycobacterial virulence genes in the genome of the emerging pathogen Mycobacterium abscessus. PloS One 2009; 4: e5660.

12 Verstovsek S, Mesa RA, Gotlib J, et al. Long-term treatment with ruxolitinib for patients with myelofibrosis: 5-year update from the randomized, double-blind, placebo-controlled, phase 3 COMFORT-I trial. J Hematol Oncol 2017; 10: 55.

13 Abidi MZ, Haque J, Varma P, et al. Reactivation of pulmonary tuberculosis following treatment of myelofibrosis with ruxolitinib. Case Rep Hematol 2016; 2016: 2389038.

14 Heine A, Held SAE, Daecke SN, et al. The JAK-inhibitor ruxolitinib impairs dendritic cell function in vitro and in vivo. Blood 2013; 122: 1192-1202.

15 Lammas DA, De Heer E, Edgar JD, et al. Heterogeneity in the granulomatous response to mycobacterial infection in patients with defined genetic mutations in the interleukin 12-dependent interferon-gamma production pathway. Int J Exp Pathol 2002; 83: 1-20. 